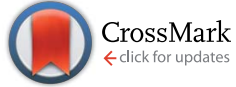

Cite this: J. Mater. Chem. C, 2014, 2 10189

Received 1st October 2014 Accepted 27th October 2014

DOI: $10.1039 / \mathrm{c} 4 \mathrm{tc0} 02218 \mathrm{a}$

www.rsc.org/MaterialsC

\section{Band convergence in the non-cubic chalcopyrite compounds $\mathrm{Cu}_{2} \mathrm{MGeSe}_{4} \dagger$}

\author{
Wolfgang G. Zeier, ${ }^{\text {*a }}$ Hong Zhu, ${ }^{\mathrm{b}}$ Zachary M. Gibbs, ${ }^{a}$ Gerbrand Ceder, ${ }^{\text {b }}$ \\ Wolfgang Tremel ${ }^{\mathrm{C}}$ and $G$. Jeffrey Snyder ${ }^{\mathrm{a}}$
}

\begin{abstract}
Inspired by recent theoretical predictions on band convergence in the tetragonal chalcopyrite compounds, we have explored the influence of the crystal structure on the transport and bandstructure of different quaternary chalcopyrites. In theory, a changing lattice parameter ratio of $c / 2 a$ towards unity should lead to band convergence due to a more cubic and higher symmetry structure. In accordance with this prediction, the different solid solutions explored in this manuscript show a significant impact on the electronic transport depending on the ratio of the lattice parameters. An increasing lattice parameter ratio results in an increase of the carrier effective masses which can be explained by converging bands, ultimately leading to an increase of the power factor and thermoelectric figure of merit in the class of non-cubic chalcopyrite compounds $\mathrm{Cu}_{2} \mathrm{MGeSe}_{4}$. However, the calculations via density functional theory show that the critical value of $c / 2 a$, where band convergence occurs, will be different from unity due to symmetry and chemical influences on the band structure.
\end{abstract}

\section{Introduction}

Thermoelectric technology is being used as a clean and efficient approach to harvest waste energy or solid-state cooling. The performance of a thermoelectric material is determined by its figure of merit $z T=\alpha^{2} \sigma T / \kappa$. Obtaining a high $z T$ requires a large Seebeck coefficient $\alpha$, a high electrical conductivity $\sigma$ and a low thermal conductivity $\kappa$. For high $z T$ the carrier concentration needs to be optimized by adjusting the doping, which is often observed along with an increase in the power factor $\alpha^{2} \sigma{ }^{1}$ High optimized $z T$ can only been obtained through a high quality factor $^{2}$ which can be increased, for example, by the convergence of highly-degenerate multi-valley electronic bands. Band convergence has been found for example in $\mathrm{Mg}_{2} \mathrm{Si}-\mathrm{Mg}_{2} \mathrm{Sn},{ }^{3,4}$ PbTe (ref. 5-10) and SnTe. ${ }^{11}$

The convergence of different valence or conduction bands at a certain temperature or chemical potential leads to an increase in the valley degeneracy (number of carrier pockets) $N_{\mathrm{V}}$, which increases the density of states effective mass $m_{\mathrm{DOS}}^{*}\left(m_{\mathrm{DOS}}^{*}=\right.$ $\left.m_{\mathrm{b}}{ }^{*}{ }_{\mathrm{V}}{ }^{2 / 3}\right){ }^{2,12}$ The band effective mass $m_{\mathrm{b}}^{*}$ is related to the carrier

\footnotetext{
${ }^{a}$ Department of Material Science and Applied Physics, California Institute of Technology, 1200 E California Blvd, Pasadena, CA 99125, USA. E-mail: zeier@ caltech.edu

${ }^{b}$ Department of Materials Science and Engineering, Massachusetts Institute of Technology, 77 Massachusetts Avenue, Cambridge, MA 02139, USA

'Institut für Anorganische und Analytische Chemie, Johannes Gutenberg-University Mainz, Duesbergweg 10-14, 55099 Mainz, Germany

$\dagger$ Electronic supplementary information (ESI) available: Calculated electronic band structures for the stannite and kesterite structure types. See DOI: $10.1039 / \mathrm{c} 4$ tc02218a
}

mobilities via the inverse relationship $\mu=e \tau / m_{\mathrm{b}}^{*}$, with the scattering relaxation time $\tau$. This increase in the density of states effective mass leads to the above mentioned increase in the quality factor of a material. ${ }^{2}$ In general, highly symmetrical crystal structures can exhibit a large band degeneracy and consequently many good thermoelectric materials have cubic

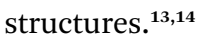

Recently, Zhang et al. have theoretically explored the possibility of band convergence in the non-cubic chalcopyrite crystal structures. ${ }^{15}$ Chalcopyrites are structurally derived from the diamond structure and each atom is coordinated by four nearest neighbors, forming tetrahedrally bonded structures. Depending on the composition, these multinary phases form binary, ternary, and quaternary compounds where the two substructures are populated with cations and anions. ${ }^{16}$ The binary compounds crystallize in the cubic-sphalerite structure type (space group $F \overline{4} 3 m$ ). Further lowering the symmetry by ordered cation substitution with two metals leads to the chalcopyrite structure type $\mathrm{A}^{\mathrm{I}} \mathrm{B}^{\mathrm{III}} \mathrm{X}_{2}{ }^{\mathrm{VI}}(\bar{I} \overline{4} d)$, with the mineral chalcopyrite $\mathrm{CuFeS}_{2}$ as the prototype. This ordered substitution doubles the translational period along the $z$-direction leading to a tetragonal crystal system. Due to different interactions between the metals and the anions, resulting in different bond lengths and bond angles, a tetragonal distortion takes place resulting in a $c / 2 a$-ratio $<1 .{ }^{17} \mathrm{~A}$ further decrease in symmetry leads to the stannite $(\overline{4} 2 \mathrm{~m}$, see Fig. 1$)$ or the kesterite type structure $(I \overline{4})$. This decrease in symmetry is achieved by substitution of elements (stannite). A further symmetry decay via metal ordering results in the kesterite structure type. The lower symmetry and larger unit cell structures provide lower 
lattice thermal conductivity, ${ }^{18}$ although the electronic structure also becomes distorted.

Due to the crystal field effect and the tetragonal distortions, ${ }^{19,20}$ the valence band splits into a non-degenerate band $\Gamma_{4 v}$ and a doubly degenerate band $\Gamma_{5 v} \cdot{ }^{15}$ Zhang et al. have theoretically predicted, that for a $c / 2 a$-ratio $<1$ the $\Gamma_{4 v}$ band is higher in energy and an increasing $c / 2 a$-ratio leads to a decrease in the energy difference $\Delta$ between $\Gamma_{4 v}$ and $\Gamma_{5 v}{ }^{15}$ The decrease in energy difference between the bands leads to full band convergence, predicted when $c / 2 a$ reaches unity, representing a cubic structure. ${ }^{15}$ Upon further increasing $c / 2 a$ the two bands diverge again, with a flipped configuration of $\Gamma_{5 v}$ having a higher energy than $\Gamma_{4 v}$ (see Fig. 1). This band convergence upon changing the lattice constant ratio leads to an increase in the power factor and an increase in the figure of merit in this class of materials.

Previous studies of these compounds have mainly focused on the intrinsic thermoelectric transport properties, doping studies or the reduction of the lattice thermal conductivities via nano crystals or phase segregations. ${ }^{21-26}$ Furthermore Zeier et al. have shown that a restructuring process in the series of solid solutions $\mathrm{Cu}_{2} \mathrm{Zn}_{1-x} \mathrm{Fe}_{x} \mathrm{GeSe}_{4}$ changes the $c / 2 a$ axis ratio, which leads to enhanced point defect scattering and a reduction in the thermal conductivity due to the high local anisotropic structural disorder. ${ }^{27}$ The changing bond angles and bond lengths in these materials strongly influence the thermal transport properties in these materials. ${ }^{27,28}$

Inspired by these recent predictions on band convergence in the tetragonal chalcopyrite compounds, we have theoretically and experimentally explored the influence of the changing $c / 2 a$ lattice parameter ratios of different compositions and solid solutions on the electronic structure and sought to identify its influence on electronic transport. While the series of solid solution which exhibits a constant $c / 2 a$ ratio does not show any changes in the effective masses, an increase in $c / 2 a$ results in an increase of the effective mass. This increasing effective mass, due to valley degeneracy, ultimately leads to an increase in the power factor and the figure of merit, corroborating a convergence of the $\Gamma_{4 v}$ and $\Gamma_{5 v}$ bands upon changing $c / 2 a$, predicted by density functional theory. However, we will show that the critical value of $c / 2 a$ for a full band convergence is not necessarily unity. Chemical and structural effects lead to different band configurations and critical $c / 2 a$ ratios at which band convergence occurs.
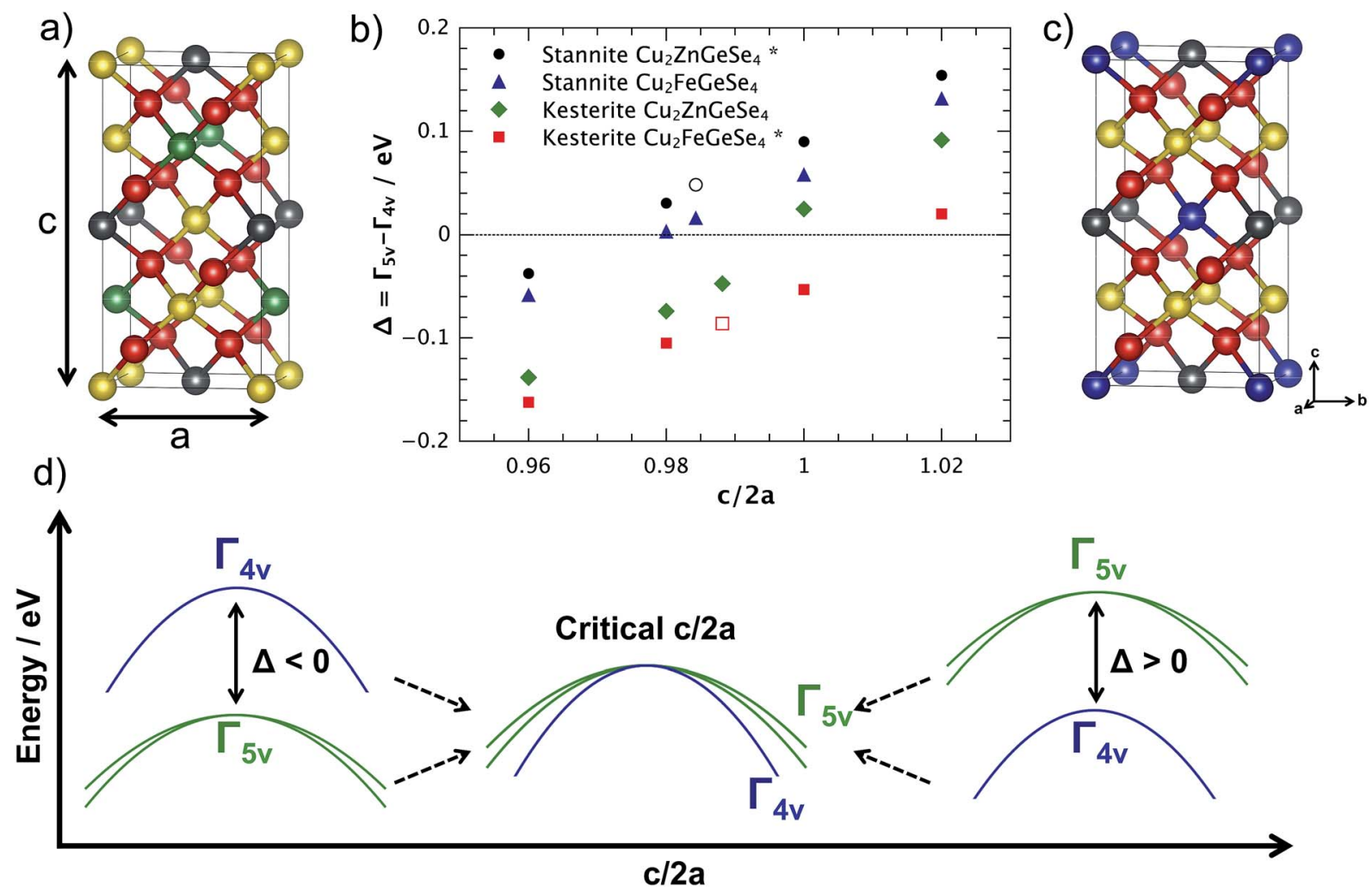

Fig. 1 Kesterite (a), space group $/ \overline{4}$, and stannite (c), space group $/ \overline{4} 2 m$, structure types of $\mathrm{Cu}_{2} \mathrm{FeGeSe}_{4}$ and $\mathrm{Cu}_{2} \mathrm{ZnGeSe} \mathrm{H}_{4}$ respectively. The red spheres represent selenium, grey germanium and yellow the copper cations. Zinc and iron are shown as blue and green spheres, respectively. (a) Shows the axis ratio, where a $c / 2 a$ of unity represents a cubic structure. (b) Shows the effect of the $c / 2 a$ ratio on the band energy difference $\Delta$ between the $\Gamma_{4 v}$ and the doubly degenerate $\Gamma_{5 v}$ valence bands. Negative values represent the situation in which $\Gamma_{4 v}$ is at higher energy than $\Gamma_{5 v}$ as seen in (d). An increasing $c / 2 a$ leads to a full band convergence $(\Delta=0)$ at a critical $c / 2 a$ value. ${ }^{15}$ With further increasing $c / 2 a$, the band configuration changes. Due to the differences in the structures and the chemical composition, different band configurations can be observed for $\mathrm{Cu}_{2} \mathrm{ZnGeSe}_{4}$ and $\mathrm{Cu}_{2} \mathrm{FeGeSe}_{4}$ at their experimentally observed $c / 2 a$ ratios (open data markers). The compositions in their existing structure types are labeled with an asterix. 


\section{Experimental}

Bulk samples of polycrystalline $\mathrm{Cu}_{2+x} \mathrm{Zn}_{1-x} \mathrm{GeSe}_{4}, \mathrm{Cu}_{2} \mathrm{Zn}_{1-x^{-}}$ $\mathrm{Fe}_{x} \mathrm{GeSe}_{4}$ and $\mathrm{Cu}_{2.025} \mathrm{Zn}_{0.975-y} \mathrm{Fe}_{y} \mathrm{GeSe}_{4}$ were prepared via solid state reactions using elemental powders of $\mathrm{Cu}$ (Alfa Aesar, 99.999\%), Zn (Sigma Aldrich, 99.995\%), Fe (Alfa Aesar, 99.998\%), Ge (Chempur, 99.99\%) and Se (Alfa Aesar, 99.999\%). Phase purity of the starting materials was verified by X-ray diffraction, and all synthetic procedures were carried out in a $\mathrm{N}_{2}$ drybox. The starting elements were thoroughly ground, sealed in quartz ampoules, and annealed in a first step for 48 hours at $923 \mathrm{~K}$ and $673 \mathrm{~K}$, for $[\mathrm{Fe}]<0.4$ and $[\mathrm{Fe}] \geq 0.4$, respectively. In a following second step, the powders were re-annealed for 96 hours at $1073 \mathrm{~K}$ and $873 \mathrm{~K}$, respectively. The annealed material was hand ground and consolidated into 1-1.5 mm thick, $12 \mathrm{~mm}$ diameter disks at $873 \mathrm{~K}$ for 5 hours under a pressure of $40 \mathrm{MPa}$ by induction hot pressing in high density graphite dies. ${ }^{29}$ The resulting samples have more than 95\% theoretical density, determined from the mass and geometry of the consolidated disks.

X-ray diffraction measurements were performed on a Siemens D5000 powder diffractometer with a Braun M50 position sensitive detector and $\mathrm{CuK} \alpha_{1}$ radiation (Ge (220) monochromator). To obtain lattice parameters, Pawley refinements were performed with TOPAS Academic V4.1, ${ }^{30}$ applying the fundamental parameter approach using the crystallographic data from Schäfer and Nitsche. ${ }^{16}$

The Seebeck coefficient was calculated from the slope of the voltage $v s$. temperature gradient measurements from Chromel$\mathrm{Nb}$ thermocouples, applying a temperature gradient of $10 \mathrm{~K} .^{31}$ Electrical resistivity, Hall coefficient, and carrier concentration were measured using the van der Pauw technique under a reversible magnetic field of $2 \mathrm{~T}$, a current of $20 \mathrm{~mA}$, and pressure-assisted contacts. ${ }^{32}$ All measurements were performed under dynamic vacuum and on multiple samples for each composition.

For a single parabolic band relationship, solutions to the Boltzmann transport equation within the relaxation time approximation were used to model the relationship between carrier concentration and the Seebeck coefficient. Complete transport modeling procedures, ${ }^{33}$ as well as diffraction and temperature dependent transport data can be found in previous publications. $^{24,27,34}$

Density functional theory (DFT) calculations with the Perdew-Burke-Ernzerhof (PBE) $)^{35,36}$ generalized gradient approximation (GGA) and projector augmented wave (PAW) ${ }^{37}$ pseudopotentials were implemented in the Vienna ab initio simulation package (VASP). ${ }^{38}$ A plane wave cutoff of $520 \mathrm{eV}$, a KPOINT mesh of $6 \times 6 \times 4$ and a ferromagnetic state for the initial magnetization of the density were used in our calculations. A HSE06 functional was employed for electronic structure computations, which has shown to improve the band gap across a wide range of materials. ${ }^{39,40}$ We adopted the experimental structures for stannite-type $\mathrm{Cu}_{2} \mathrm{ZnGeSe}_{4}$ and kesterite-type $\mathrm{Cu}_{2} \mathrm{FeGeSe}_{4}$ within our calculations. To understand the effect of $c / 2 a$ on the convergence of $\Gamma_{5 v}$ and
$\Gamma_{4 v}$, we have rigidly varied the $c$ lattice parameter. We have also looked into the band structure of these two materials at experimental lattice parameter but with a first-principles ionic relaxation, which displays similar band convergence but a larger band gap, as compared to the computed band structures without atomic relaxation. Complete electronic band structures for stannite-type $\mathrm{Cu}_{2} \mathrm{ZnGeSe}_{4}$ and kesteritetype $\mathrm{Cu}_{2} \mathrm{FeGeSe}_{4}$ can be found in the ESI $\dagger$ and have been published elsewhere. ${ }^{27}$

\section{Results and discussion}

\subsection{Structural considerations}

The refined ratio $c / 2 a$ of the lattice parameters for the solid solutions $\mathrm{Cu}_{2+x} \mathrm{Zn}_{1-x} \mathrm{GeSe}_{4}, \mathrm{Cu}_{2} \mathrm{Zn}_{1-x} \mathrm{Fe}_{x} \mathrm{GeSe}_{4}$ and $\mathrm{Cu}_{2.025^{-}}$ $\mathrm{Zn}_{0.975-y} \mathrm{Fe}_{y} \mathrm{GeSe}_{4}$ can be found in Fig. 2. The substitution of $\mathrm{Zn}$ with $\mathrm{Fe}$ results in a non-Vegard like behavior with a maximum of the lattice parameter ratio at $70 \% \mathrm{Fe}$ content, due to a 3-stage cation restructuring process which has been previously reported for $\mathrm{Cu}_{2} \mathrm{Zn}_{1-x} \mathrm{Fe}_{x} \mathrm{GeSe}_{4} \cdot{ }^{27}$ The end members $\mathrm{Cu}_{2} \mathrm{ZnGeSe}_{4}$ and $\mathrm{Cu}_{2} \mathrm{FeGeSe}_{4}$ crystallize in the stannite and kesterite structure type (Fig. 1), respectively. ${ }^{27}$ The equal crystal radii of the cations
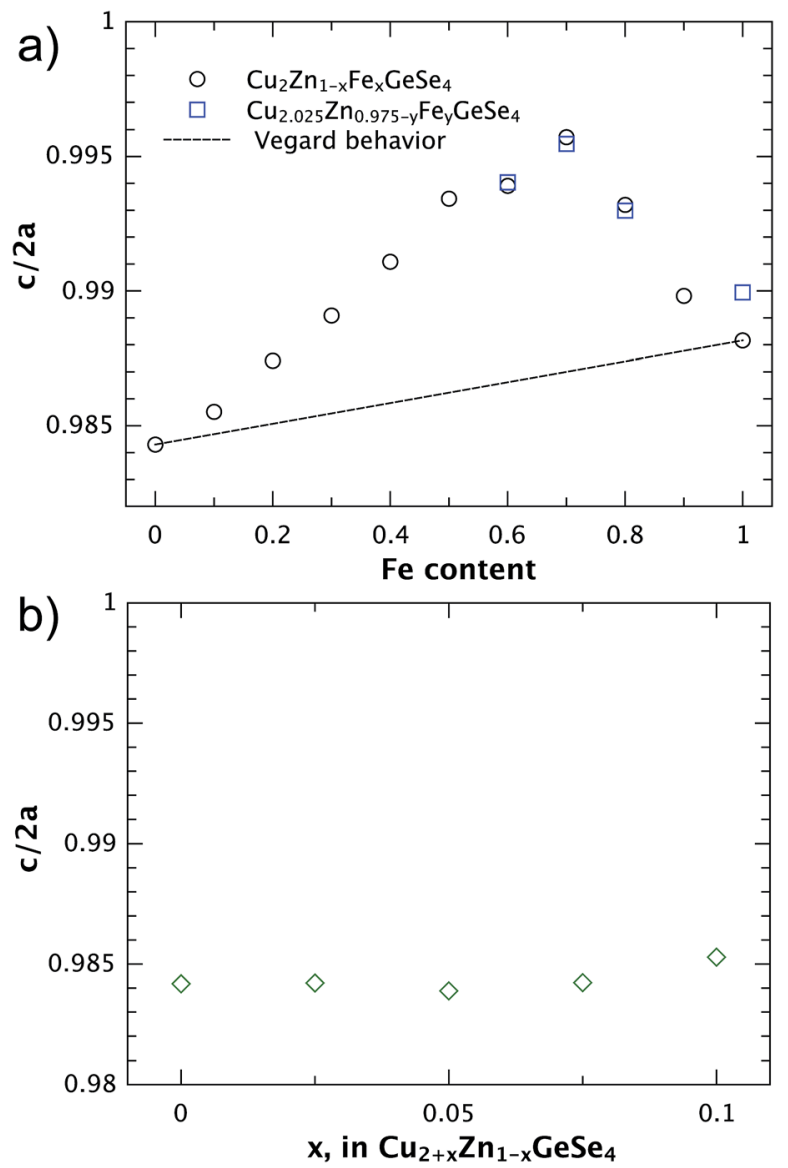

Fig. 2 (a) Shows the axis ratio $c / 2 a$ of the solid solutions $\mathrm{Cu}_{2} \mathrm{Zn}_{1-x^{-}}$ $\mathrm{Fe}_{x} \mathrm{GeSe}_{4}$ and $\mathrm{Cu}_{2.025} \mathrm{Zn}_{0.975-y} \mathrm{Fe}_{y} \mathrm{GeSe}_{4}$. A restructuring process leads to an elongation of the $c$-axis, ${ }^{27}$ leading to a non-Vegard like behavior. (b) Constant $c / 2 a$ for $\mathrm{Cu}_{2+x} \mathrm{Zn}_{1-x} \mathrm{GeSe}_{4}$ 
$\mathrm{Cu}^{+}$and $\mathrm{Zn}^{2+}(0.74 \mathrm{pm} \text { in tetrahedral coordination })^{41}$ lead to a constant lattice parameter ratio in the solid solution $\mathrm{Cu}_{2+x^{-}}$ $\mathrm{Zn}_{1-x} \mathrm{GeSe}_{4}$ as seen in Fig. 2. Combining the transport properties of two solid solutions, which exhibit changing lattice parameter ratios, and one which does not exhibit any changes are a good starting point to compare and analyze the effects of the lattice parameter ratios on the electronic transport. Therefore, we will use the example of $\mathrm{Cu}_{2+x} \mathrm{Zn}_{1-x} \mathrm{GeSe}_{4}$ as a baseline to compare other solid solutions where the $c / 2 a$ ratio changes and approaches unity.

\subsection{Theoretical predictions}

Fig. 1 shows the calculated energy difference $\Delta$ between the $\Gamma_{4 v}$ and the doubly degenerate $\Gamma_{5 v}$ valence bands of the stannite $\left(\mathrm{Cu}_{2} \mathrm{ZnGeSe}_{4}\right)$ and the kesterite $\left(\mathrm{Cu}_{2} \mathrm{FeGeSe}_{4}\right)$ structure types. Positive values of $\Delta$ represent the case where the doubly degenerate $\Gamma_{5 v}$ band is located at higher energies than $\Gamma_{4 v}$. Full band convergence occurs when the energy difference, $\Delta$, becomes zero or effectively within $2 k_{\mathrm{B}} T$. As predicted by Zhang et al. ${ }^{15}$ changing the $c / 2 a$ ratio affects the position of the two valence bands. However, differences in $c / 2 a$ values in the compositions of $\mathrm{Cu}_{2} \mathrm{ZnGeSe}_{4}$ and $\mathrm{Cu}_{2}$ $\mathrm{FeGeSe}_{4}$ can be observed. Band convergence is obtained at different values of $c / 2 a$ for the two compounds $\mathrm{Cu}_{2} \mathrm{ZnGeSe}_{4}$ and $\mathrm{Cu}_{2} \mathrm{FeGeSe}_{4}$ and convergence is not exactly at $c / 2 a=1$. While the small deviations from unity, are within the estimated uncertainty by Zhang et al. ${ }^{15}$ the stannite structure $\mathrm{Cu}_{2} \mathrm{ZnGeSe}_{4}$ exhibits band convergence $(\Delta=0)$ at a critical $c /$ $2 a$ value below unity. In the kesterite type $\mathrm{Cu}_{2} \mathrm{FeGeSe}_{4}$ however, band convergence is achieved at $c / 2 a>1$. While the deviations from unity are small, all experimentally accessible lattice parameters of these solid solutions are located in a very narrow range of $c / 2 a$, between 0.98 and 1 . Indeed, at the experimentally observed lattice parameter values for $\mathrm{Cu}_{2}$ $\mathrm{FeGeSe}_{4}$ and $\mathrm{Cu}_{2} \mathrm{ZnGeSe}_{4}$, the valence bands $\Gamma_{4 v}$ and $\Gamma_{5 v}$ are at different energetic configurations (see Fig. 1), i.e. negative and positive $\Delta$. While $\Gamma_{4 v}$ is located at higher energies than $\Gamma_{5 v}$ in the case of kesterite- $\mathrm{Cu}_{2} \mathrm{FeGeSe}_{4}$, the situation is reversed for stannite- $\mathrm{Cu}_{2} \mathrm{ZnGeSe}_{4}$. In order to assess the reasons for this flipped electronic band configuration, calculations were also performed on $\mathrm{Cu}_{2} \mathrm{FeGeSe}_{4}$ in a hypothetical stannite structure type and for $\mathrm{Cu}_{2} \mathrm{ZnGeSe}_{4}$ as kesterite (see Fig. 1). The change in structure type shifts the critical ratio of $c / 2 a$ significantly. In addition, a further difference in the band energies between stannite- $\mathrm{Cu}_{2} \mathrm{FeGeSe}_{4}$ and stannite- $\mathrm{Cu}_{2} \mathrm{ZnGeSe}_{4}$ within the same structure type, due to the differences of the Fe and $\mathrm{Zn}$ atomic orbitals, can also be observed leading to shifts in the critical ratio of $c / 2 a$. The symmetry differences due to the structure types and the chemical difference between $\mathrm{d}^{10}-\mathrm{Zn}^{2+}$ and $\mathrm{d}^{6}-\mathrm{Fe}^{2+}$, both vary the predicted band structures and the critical $c / 2 a$ values for band convergence diverge from unity. Due to these structure and chemistry dependence, it is important to assess the valence band configuration of these chalcopyrite compounds before targeted altering of the $c / 2 a$ ratio is attempted, when already within 0.02 of unity.

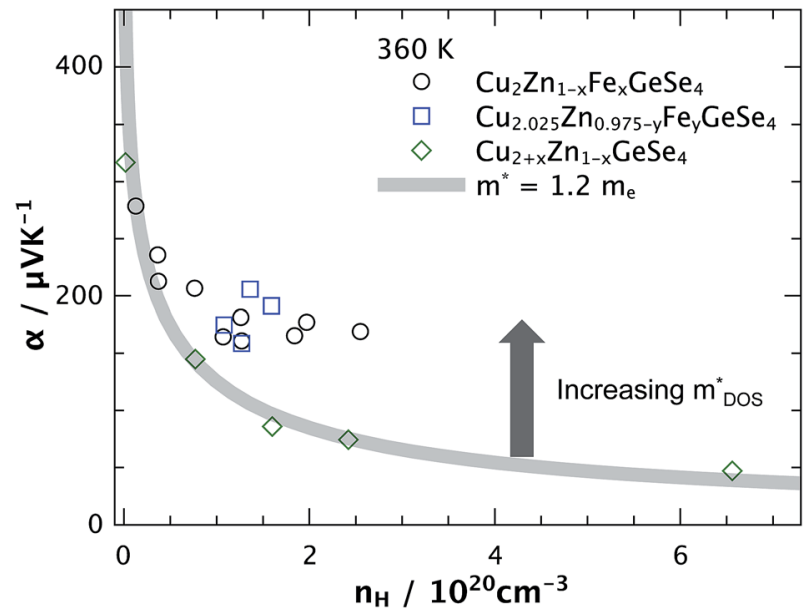

Fig. 3 Pisarenko relation of the solid solutions $\mathrm{Cu}_{2+x} \mathrm{Zn}_{1-x} \mathrm{GeSe}_{4}{ }^{24}$ $\mathrm{Cu}_{2} \mathrm{Zn}_{1-x} \mathrm{Fe}_{x} \mathrm{GeSe}_{4}{ }^{34}$ and $\mathrm{Cu}_{2.025} \mathrm{Zn}_{0.975-y} \mathrm{Fe}_{y} \mathrm{GeSe}_{4}$ at $360 \mathrm{~K}$. The gray line was generated using a single parabolic band approximation and an effective mass of $1.2 \mathrm{~m}_{\mathrm{e}}$. No change of the carrier effective mass can be seen in the solid solution of $\mathrm{Cu}_{2+x} \mathrm{Zn}_{1-x} \mathrm{GeSe}_{4},{ }^{24}$ which exhibits a constant $c / 2 a$. The increasing $c / 2 a$ of the other series of solid solutions leads to an increase of the effective mass and hence an increase in $\alpha$.

\subsection{Electronic transport}

Measured Seebeck coefficients $\alpha$ of all compositions versus their Hall carrier concentrations $n_{\mathrm{H}}$ can be found in Fig. 3. Due to the reported phase transformation at higher temperatures, ${ }^{42}$ all measurement data are shown at $360 \mathrm{~K}$. All Seebeck coefficients and carrier concentrations are positive, showing holes as the predominant carriers in these p-type semiconducting materials. Measured Hall carrier concentrations of the holes range between 2-5 $\mathrm{cm}^{2} \mathrm{~V}^{-1} \mathrm{~s}^{-1}$ and have been reported elsewhere. ${ }^{24,27,34}$ For the solid solution of $\mathrm{Cu}_{2+x} \mathrm{Zn}_{1-x} \mathrm{GeSe}_{4}$ an

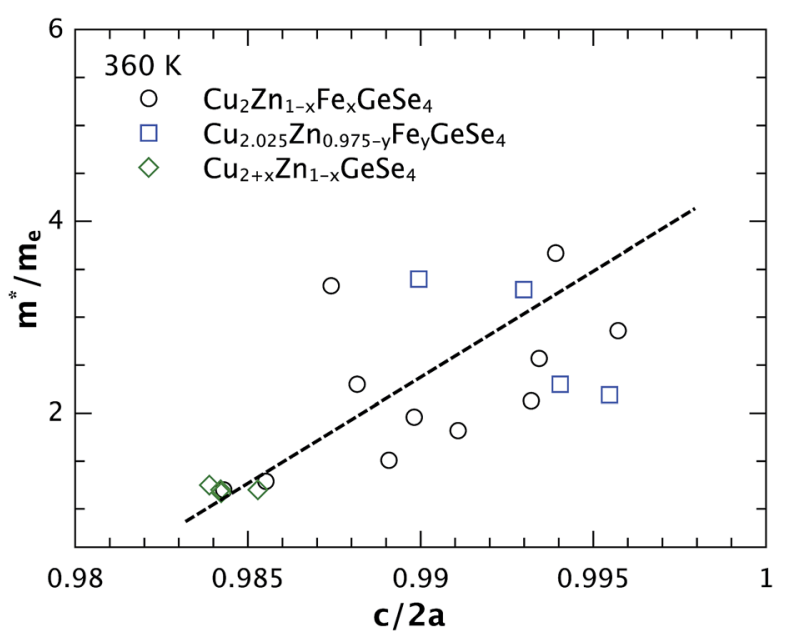

Fig. 4 Density of states effective mass $m^{*}$, obtained at $360 \mathrm{~K}$ using a single parabolic band approximation, versus the $c / 2 a$ ratios of the different solid solutions. The increasing effective mass with increasing $c / 2 a$ ratio suggests a band convergence behavior, which increases $m^{*}$ obtained from the Seebeck coefficients. The broken line designates a constant increase of $m^{*}$. 

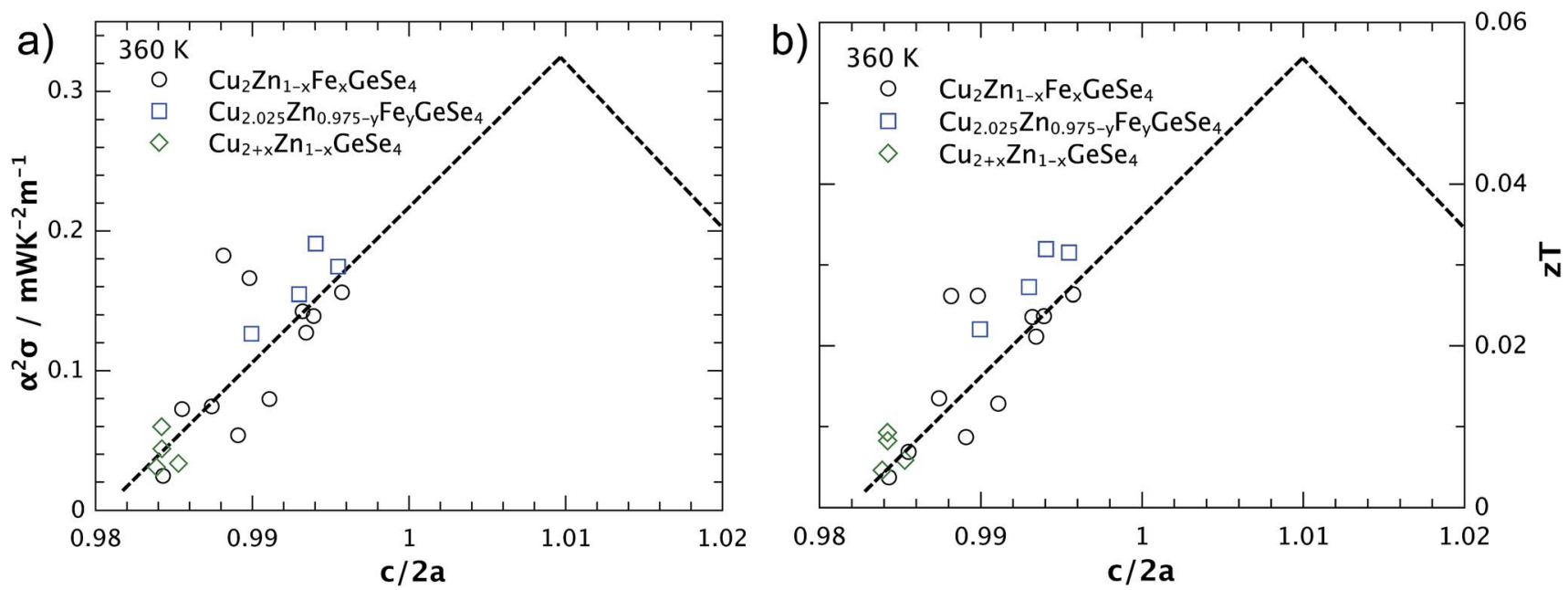

Fig. 5 (a) Power factors $\alpha^{2} \sigma$ and (b) figure of merit $z T$ of $\mathrm{Cu}_{2} \mathrm{Zn}_{1-x} \mathrm{Fe}_{x} \mathrm{GeSe}_{4}$ and $\mathrm{Cu}_{2.025} \mathrm{Zn}_{0.975-y} \mathrm{Fe}_{y} \mathrm{GeSe}_{4}$ are increasing with increasing $c / 2 a$ ratio. The broken lines serve as a guide to the eye, with a maximum power factor and $z T$ for a critical $c / 2 a$-ratio $>1$, as obtained from band structure calculations for $\mathrm{Cu}_{2} \mathrm{FeGeSe}_{4}$.

effective mass $m^{*}$ of $1.2 m_{\mathrm{e}}$ was used to calculate the grey line in Fig. 3. The data fall near or on the modeled curve showing that the single parabolic band (single $m^{*}$ ) is a good starting model for the electronic transport. The substitution of $\mathrm{Zn}^{2+}$ with $\mathrm{Cu}^{+}$ does not significantly change the effective mass of the holes nor does it result in any changes of the $c / 2 a$ ratio. While the effective mass remains constant for $\mathrm{Cu}_{2+x} \mathrm{Zn}_{1-x} \mathrm{GeSe}_{4}$, the compositions which exhibit different $c / 2 a$ ratios (Fig. 2) show an increase in the effective mass with increasing $c / 2 a$ ratio. A possible band convergence at higher $c / 2 a$ ratios would result in a higher valley degeneracy $N_{\mathrm{V}}$, resulting in a higher density of states effective mass $m_{\mathrm{DOS}}^{*}$ (eqn (2)). The Seebeck coefficient $\alpha$ of a degenerate semiconductor with a parabolic band dispersion and lattice deformation (phonon) scattering is given by: ${ }^{1,2}$

$$
\alpha=\frac{8 \pi^{2} k_{\mathrm{B}}{ }^{2}}{3 e h^{2}} m_{\mathrm{DOS}}^{*} T\left(\frac{\pi}{3 n}\right)^{2 / 3},
$$

and

$$
m_{\mathrm{DOS}}^{*}=m_{\mathrm{b}}^{*} N_{\mathrm{V}}^{2 / 3} .
$$

An increase in the density of states effective mass would hence ultimately result in an increase of the Seebeck coefficient. Therefore, extracting the effective masses from the carrier concentration, $n$, dependent Seebeck coefficients provides a way to assess possible changes in the valley degeneracy. Fig. 4 shows the density of states effective masses of the solid solutions $\mathrm{Cu}_{2} \mathrm{Zn}_{1-x} \mathrm{Fe}_{x} \mathrm{GeSe}_{4}$ and $\mathrm{Cu}_{2.025} \mathrm{Zn}_{0.975-y} \mathrm{Fe}_{y} \mathrm{GeSe}_{4}$ and their dependence on the lattice parameter ratios. With increasing ratio of $c / 2 a$ the effective mass increases, due to a convergence of the $\Gamma_{4 v}$ and $\Gamma_{5 v}$ bands, as predicted by Zhang et al. ${ }^{15}$ This behavior cannot be seen in the solid solution $\mathrm{Cu}_{2+x} \mathrm{Zn}_{1-x} \mathrm{GeSe}_{4}$, because the $c / 2 a$ ratio remains constant, showing a clear indication that the ratio of the lattice parameters does indeed influence the respective position of the bands. With increasing $c / 2 a$ ratio, the energy difference between $\Gamma_{4 v}$ and $\Gamma_{5 v}$ decreases (see Fig. 1), effectively leading to an increase in the valley degeneracy. While DFT calculations in this work (see Fig. 1) show band offsets around $0.1 \mathrm{eV}$ for $c / 2 a \sim 0.985$ (the kesterite$\mathrm{Cu}_{2} \mathrm{FeGeSe}_{4}$ experimental value), it is important to note that some carrier population does probably exist in both the $\Gamma_{4}$ and $\Gamma_{5}$ bands because at $360 \mathrm{~K}$, this offset is around $\sim 3-4 k_{\mathrm{B}} T$. We still, however, expect the power factor to increase due to valley degeneracy as $c / 2 a$ increases because the bands move closer to "effective convergence" which is closer to 1-2 $k_{\mathrm{B}} T$.

This increasing band convergence leads to an increase in the power factor with increasing $c / 2 a$ ratios (see Fig. 5a). In the kesterite type $\mathrm{Cu}_{2} \mathrm{FeGeSe}_{4}$, band convergence should be obtained at a critical value of $c / 2 a$ above unity. The combination of increasing power factor, due a valence band convergence, and the lower lattice thermal conductivity, due to point defect scattering, ${ }^{27}$ leads to an increase of the figure of merit with increasing $c / 2 a$ (see Fig. $5 \mathrm{~b}$ ).

\section{Conclusion}

In summary, we have shown the influence of the ratio of the lattice parameters in the non-cubic chalcopyrite compounds $\mathrm{Cu}_{2} \mathrm{MGeSe}_{4}$ on the electronic transport properties, providing experimental evidence for the predicted convergence of the valence bands. The valence bands $\Gamma_{4 v}$ and $\Gamma_{5 v}$, which split due to crystal field effects, converge at a critical $c / 2 a$ ratio. Structural and chemical influences affect the convergence of the bands, leading to differences in the valence band energy configurations and the value of $c / 2 a$, where the band convergence occurs. It is therefore important to assess the valence band configuration of these chalcopyrite compounds, before targeted altering of the $c / 2 a$ ratio is attempted. This convergence of the bands leads to higher density of states effective masses, and concurrently higher power factors and figure of merits, making this a promising approach to obtain better thermoelectric performances in non-cubic systems. 


\section{Acknowledgements}

Financial support through the Excellence Initiative (DFG/GSC 266) and the Carl-Zeiss foundation is acknowledged by W.G.Z. and W.T. The theory and band structure calculations for this project were performed under the Materials Project work, supported by Department of Energy Basic Energy Sciences program under Grant no. EDCBEE, DOE Contract DE-AC02-05CH11231.

\section{References}

1 G. J. Snyder and E. S. Toberer, Nat. Mater., 2008, 7, 105-114.

2 Y. Pei, H. Wang and G. J. Snyder, Adv. Mater., 2012, 24, 61246135.

3 V. K. Zaitsev, E. N. Tkalenko and E. N. Nikitin, Sov. Phys. Solid State, 1969, 11, 221-224.

4 W. Liu, X. Tan, K. Yin, H. Liu, X. Tang, J. Shi, Q. Zhang and C. Uher, Phys. Rev. Lett., 2012, 108, 166601.

5 Y. Pei, X. Shi, A. LaLonde, H. Wang, L. Chen and G. J. Snyder, Nature, 2011, 473, 66-69.

6 Y. Pei, N. A. Heinz, A. LaLonde and G. J. Snyder, Energy Environ. Sci., 2011, 4, 3640-3645.

7 A. D. LaLonde, Y. Pei, H. Wang and G. Jeffrey Snyder, Mater. Today, 2011, 14, 526-532.

8 Y. Pei, A. LaLonde, S. Iwanaga and G. J. Snyder, Energy Environ. Sci., 2011, 4, 2085-2089.

9 Z. M. Gibbs, H. Kim, H. Wang, R. L. White, F. Drymiotis, M. Kaviany and G. Jeffrey Snyder, Appl. Phys. Lett., 2013, 103, 262109.

10 L. D. Zhao, H. J. Wu, S. Q. Hao, C. I. Wu, X. Y. Zhou, K. Biswas, J. Q. He, T. P. Hogan, C. Uher, C. Wolverton, V. P. Dravid and M. G. Kanatzidis, Energy Environ. Sci, 2013, 6, 3346-3355.

11 A. Banik and K. Biswas, J. Mater. Chem. A, 2014, 2, 96209625.

12 Y. Pei, A. D. LaLonde, H. Wang and G. J. Snyder, Energy Environ. Sci., 2012, 5, 7963-7969.

13 G. D. Mahan and J. O. Sofo, Proc. Natl. Acad. Sci. U. S. A., 1996, 93, 7436-7439.

14 F. J. DiSalvo, Science, 1999, 285, 703-706.

15 J. Zhang, R. Liu, N. Cheng, Y. Zhang, J. Yang, C. Uher, X. Shi, L. Chen and W. Zhang, Adv. Mater., 2014, 26, 3848-3853.

16 W. Schäfer and R. Nitsche, Mater. Res. Bull., 1974, 9, 645654.

17 S. Schorr, Thin Solid Films, 2007, 515, 5985-5991.

18 E. S. Toberer, A. Zevalkink and G. J. Snyder, J. Mater. Chem., 2011, 21, 15843-15852.

19 J. Rowe and J. Shay, Phys. Rev. B: Solid State, 1971, 3, 451.

20 L. Artus, Y. Bertrand and C. Ance, J. Phys. C: Solid State Phys., 1986, 19, 5937-5943.
21 X. Y. Shi, F. Q. Huang, M. L. Liu and L. D. Chen, Appl. Phys. Lett., 2009, 94, 122103.

22 M.-L. Liu, I.-W. Chen, F.-Q. Huang and L.-D. Chen, Adv. Mater., 2009, 21, 3808-3812.

23 M.-L. Liu, F.-Q. Huang, L.-D. Chen and I.-W. Chen, Appl. Phys. Lett., 2009, 94, 202103.

24 W. G. Zeier, A. LaLonde, Z. M. Gibbs, C. P. Heinrich, M. Panthöfer, G. J. Snyder and W. Tremel, J. Am. Chem. Soc., 2012, 134, 7147-7154.

25 M. Ibáñez, D. Cadavid, R. Zamani, N. García-Castello, V. Izquierdo-Roca, W. Li, A. Fairbrother, J. D. Prades, A. Shavel, J. Arbiol, A. Perez-Rodriguez, J. R. Morante and A. Cabot, Chem. Mater., 2012, 24, 562-570.

26 M. Ibáñez, R. Zamani, A. LaLonde, D. Cadavid, W. Li, A. Shavel, J. Arbiol, J. R. Morante, S. Gorsse, G. J. Snyder and A. Cabot, J. Am. Chem. Soc., 2012, 134, 4060-4063.

27 W. G. Zeier, Y. Pei, G. Pomrehn, T. Day, N. A. Heinz, C. P. Heinrich, G. J. Snyder and W. Tremel, J. Am. Chem. Soc., 2013, 135, 726-732.

28 C. P. Heinrich, T. W. Day, W. G. Zeier, G. Je and W. Tremel, J. Am. Chem. Soc., 2014, 136, 442-448.

29 A. D. LaLonde, T. Ikeda and G. J. Snyder, Rev. Sci. Instrum., 2011, 82, 025104.

30 A. Coelho, TOPAS Academic V4.1, 2004.

31 S. Iwanaga, E. S. Toberer, A. LaLonde and G. J. Snyder, Rev. Sci. Instrum., 2011, 82, 063905.

32 K. A. Borup, E. S. Toberer, L. D. Zoltan, G. Nakatsukasa, J.-P. Fleurial, B. B. Iversen and G. J. Snyder, Rev. Sci. Instrum., 2012, 83, 123902.

33 A. F. May, J.-P. Fleurial and G. Snyder, Phys. Rev. B: Condens. Matter Mater. Phys., 2008, 78, 125205.

34 W. G. Zeier, T. Day, E. Schechtel, G. J. Snyder and W. Tremel, Funct. Mater. Lett., 2013, 06, 1340010.

35 J. P. Perdew, K. Burke and M. Ernzerhof, Phys. Rev. Lett., 1996, 77, 3865.

36 J. P. Perdew, K. Burke and M. Ernzerhof, Phys. Rev. Lett., 1997, 78, 1396.

37 P. Blöchl, Phys. Rev. B: Condens. Matter Mater. Phys., 1994, 50, 17953.

38 G. Kresse and J. Furthmuller, Phys. Rev. B: Condens. Matter Mater. Phys., 1996, 54, 11169.

39 J. Heyd, J. E. Peralta, G. E. Scuseria and R. L. Martin, J. Chem. Phys., 2005, 123, 174101.

40 R. Ramprasad, H. Zhu, P. Rinke and M. Scheffler, Phys. Rev. Lett., 2012, 108, 066404.

41 R. D. Shannon, Acta Crystallogr., Sect. A: Cryst. Phys., Diffr., Theor. Gen. Crystallogr., 1976, 32, 751-767.

42 W. G. Zeier, C. P. Heinrich, T. Day, C. Panithipongwut, G. Kieslich, G. Brunklaus, G. J. Snyder and W. Tremel, J. Mater. Chem. A, 2014, 2, 1790-1794. 